Pacific Journal of Mathematics

A THREE POINT CONDITION FOR SURFACES OF CONSTANT 


\title{
A THREE POINT CONDITION FOR SURFACES OF CONSTANT MEAN CURVATURE
}

\author{
EAMON B. BARRETT
}

Let $\phi(x, y)$ be a solution to the equation:

$$
\left(1+\phi_{y}^{2}\right) \phi_{x x}-2 \phi_{x} \phi_{y} \phi_{x y}+\left(1+\phi_{x}^{2}\right) \phi_{y y}=2 H\left(1+\phi_{x}^{2}+\phi_{y}^{2}\right)^{3 / 2} \text {. }
$$

The quantity $H$ in equation (1) represents the mean curvature of the surface $z=\phi(x, y)$. In case $H=0,(1)$ is the minimal surface equation. For minimal surfaces, the wellknown three point condition may be stated as follows:

Theorem 1. Let $\phi(x, y)$ be a solution to the Dirichlet problem for the minimal surface equation in some bounded region $R$. Let $T$ be the continuous space curve defined by the values of $\phi(x, y)$ over $\partial R$, the boundary of $R$. Then, if $P$ is a plane tangent to the surface $z=\phi(x, y)$ for $(x, y)$ in $R, P$ will have at least 4 points in common with $T$.

The objective of this paper is to establish a natural analogue of the three-point condition for surfaces of positive, constant mean curvature.

It will be shown that certain "interior tangent spheres" of radius $1 / H$ play the same roles, for surfaces of constant mean curvature $H>0$ defined on a disk of radius $\rho<1 / H$, that tangent planes do for minimal surfaces.

(Rado's statement of the three-point condition for minimal surfaces appears in [3], pg. 34, et. seq.)

DEFINITION. Let $S$ be a surface of constant mean curvature $H>0$, defined by $z=\phi(x, y)$, where $\phi(x, y)$ is a solution to equation (1) in some region $R$. At the point $\bar{x}_{0}=\left(x_{0}, y_{0}, \phi\left(x_{0}, y_{0}\right)\right.$, let the normal line to the surface be drawn. Let a sphere, $P_{0}$, of radius $1 / H$ be constructed, whose center lies a distance $1 / H$ from $\bar{x}_{0}$, on the normal line through $\bar{x}_{0}$, in the direction specified by the normal vector to $S$ at $\bar{x}_{0} . \quad P_{0}$ will be called the "interior tangent sphere to $S$ at $\bar{x}_{0}$ ".

In case $R$ is a disk of radius $\rho<1 / H$, and $T$ is a space curve consisting of the (continuous) boundary values of $\phi(x, y)$ on $\partial R$, it will be shown that $P_{0}$ has at least four points in common with $T$ :

Theorem II. Let $P_{0}$ be the interior tangent sphere to the surface $z=\phi(x, y)$ at a non-umbilical point, $x_{0}$, where $\dot{\phi}(x, y)$ is a solution to a given Dirichlet problem for equation (1) in the disk $x^{2}+y^{2}=\rho^{2}<1 / H^{2}$, $H>0$. Let $T$ be the continuous space curve defined by the values of $\phi(x, y)$ over the boundary of the disk. Then $P_{0}$ must have at least 
4 points in common with $T$.

The assumption that $\bar{x}_{0}$ is a non-umbilical point is not severe, since as in the case of minimal surfaces, the umbilical points of a surface of constant mean curvature are isolated points, except for the sphere, for which every point is umbilical. The proof of Theorem II will depend on the observation that the difference of two solutions to equation (1) must satisfy the strong maximum principle. (Ref. R. Courant and D. Hilbert [1] and on a comparison lemma due to $R$. Finn (Ref. [2].)

As $H \rightarrow 0$, the radius of the "interior tangent sphere" of Theorem II tends towards infinity, and Theorem II becomes a statement of the three point condition for minimal surfaces.

Much of the material in this paper was contained in the author's doctoral thesis, written at Stanford University under the direction of Robert Finn and Newton Hawley. The author wishes to thank Professors Finn and Hawley for their advice and encouragement.

2. Comparison theorems for quasi-linear elliptic equations. In this section we will state, without proof, several theorems for quasi-linear elliptic equations which are essential for the proof of the three-point condition for surfaces of constant mean curvature.

The first is the maximum principle for quasi-linear equations having the form of equation (1).

$$
\text { Let } M(\phi)=\left(1+\phi_{y}^{2}\right) \phi_{x x}-2 \phi_{x} \phi_{y} \phi_{x y}+\left(1+\phi_{x}^{2}\right) \phi_{y y} ;
$$

THEOREM 2.1. (Courant-Hilbert, [1], pg. 321, et. seq.). If $\phi(x, y)$ is $C^{2}$ in a domain $R$, and if $\phi(x, y)$ has a maximum at an interior point of $R$, then $M(\phi) \leqq 0$ at this point.

Theorem 1 implies that solutions of equation (1) in $R$ will not have interior maxima. Interior minima may exist, however, as with the hemispherical solution

$$
\phi(x, y)=-\sqrt{1 / H^{2}-\left(x^{2}+y^{2}\right)} .
$$

Different solutions of the same quasi-linear equation may be compared, as stated in the following comparison theorem (Courant-Hilbert, [1], pg. 322 et seq.):

THEOREM 2.2. Let $\phi$ and $\Psi$ be solutions of equation (1) in some region $R$. Denote by $\omega$ the difference of these solutions, $\omega=\phi-\psi$. Then $\omega$ has neither a maximum nor a minimum in the interior of $R$. 
A theorem concerning the comparison of solutions of quasi-linear elliptic equations, which is of importance in the study of minimal surfaces and surfaces of constant mean curvature has been proved by R. Finn (R. Finn, [2]).

\section{THEOREM 2.3.}

Hypotheses:

(i) Let $Q(\dot{\phi})$ denote the expression:

$$
\begin{aligned}
& \sum_{i, j=1}^{2} a_{i j}\left(x_{1}, x_{2}, \dot{\phi}_{x_{1}}, \dot{\phi}_{x_{2}}\right) \dot{\phi}_{x_{i} x_{j}} \\
& +b\left(x_{1}, x_{2}, \dot{\phi}_{x_{1}}, \phi_{x_{2}}\right), a_{12}=a_{21}
\end{aligned}
$$

Assume that $\left(a_{i j}\right)$ is positive definite for $(x, y)$ in $R$ and for all $\phi(x, y)$ to be considered.

(ii) Let $\Gamma=\partial R$ be the union of two closed sets, $\Gamma_{\alpha}$ and $\Gamma_{\beta}$. Let every interior point $x_{\alpha}$ of $\Gamma_{\alpha}$ be the end point of a line segment entering $R$, and let $(\partial \phi / \partial s)$ denote the derivative of $\phi$ along this line segment, in the direction approaching $x_{\alpha}$.

(iii) Let $\phi^{(1)}$ be a function defined in $R$, such that $\phi^{(1)}$ tends to a finite or infinite limit at each point of $\Gamma_{\alpha}$, and such that

$$
\lim _{x \rightarrow \Gamma_{\alpha}}\left(\frac{\partial}{\partial s} \phi^{(1)}\right)=\infty
$$

on each of the indicated line segments.

Conclusion. Let $\dot{\phi}^{(2)}$ be any function defined and continuously differentiable in $R+\Gamma$, such that

$$
\lim _{x \rightarrow \Gamma_{\beta}}\left[\phi^{(1)}-\dot{\phi}^{(2)}\right] \geqq 0 \text {. }
$$

If $Q\left(\phi^{(1)}\right) \leqq Q\left(\phi^{(2)}\right)$ in $R$, then

$$
\lim _{x \rightarrow \Gamma_{\alpha}}\left(\phi^{(1)}-\dot{\phi}^{(2)} \geqq 0,\right.
$$

and the strict inequality holds for any approach to an interior point of $\Gamma_{\alpha}$.

Theorem 2.3 has been used by R. Finn to provide a simple proof of the fact that isolated singularities of solutions to the minimal surface equation are removable.

3. Proof of Theorem II. Let $\phi(x, y)$ be a solution to equation (1) in a region $R$, and let $P_{0}$ be the interior tangent sphere to the surface $z=\phi(x, y)$ at the point $\vec{x}_{0}=\left(x_{0}, y_{0}, \dot{\phi}\left(x_{0}, y_{0}\right)\right)$, where $\left(x_{0}, y_{0}\right)$ is 
an interior point of $R$. We shall assume that $\vec{x}_{0}$ is not an umbilical point of the surface.

Define

$$
\begin{aligned}
& p_{0}=\left.\frac{\partial \dot{\phi}}{\partial x}\right|_{x_{0}, y_{0}}, q_{0}=\left.\frac{\partial \dot{\phi}}{\partial y}\right|_{x_{0}, y_{0}}, \\
& w_{0}=1+p_{0}^{2}+q_{0}^{2} . \\
& \vec{n}_{0}=\left(-p_{0} / \sqrt{w_{0}},-q_{0} / \sqrt{w_{0}}, 1 / \sqrt{w_{0}}\right) .
\end{aligned}
$$

The center of the interior tangent sphere $P_{0}$ is located at the point

$$
\vec{x}_{0}+\frac{1}{H} \vec{n}_{0}=\left(x_{0}^{\prime}, y_{0}^{\prime}, z_{0}^{\prime}\right)
$$

The lower hemisphere of $P_{0}$ has the equation

$$
z_{0 L}(x, y)=z_{0}^{\prime}-\bigvee \overline{1 / H^{2}-\left(x-x_{0}^{\prime}\right)^{2}-\left(y-y_{0}^{\prime}\right)^{2}} .
$$

We define the difference function

$$
\Psi_{0}(x, y)=\phi(x, y)-z_{o L}(x, y) \text {. }
$$

Let $K$ denote the Gauss curvature of the surface $z=\dot{\phi}(x, y)$.

LEMMA 3.1.

$$
\left(\Psi_{0 x y}\right)^{2}-\Psi_{0 x x} \Psi_{0 y y}=w_{0}^{2}\left(H^{2}-K\right) \text { at the point }\left(x_{0}, y_{0}\right) \text {. }
$$

Proof. The lemma is established by an easy calculation, together with the observation that $\Psi_{0}\left(x_{0}, y_{0}\right)=0=\left.\Psi_{0 x}\right|_{x_{0} y_{0}}=\left.\Psi_{0 y}\right|_{x_{0} y_{0}}$, since $P_{0}$ is tangent to the surface $z=\phi(x, y)$ at the point $\vec{x}_{0}$.

Since $\vec{x}_{0}$ is not an umbilical point, $H^{2}-K>0$, and $\left(x_{0}, y_{0}, \Psi_{0}\left(x_{0}\right.\right.$, $\left.y_{0}\right)$ ) is therefore a hyperbolic point for the surface $z=\Psi_{0}(x, y)$. We shall use the familiar properties of hyperbolic points on $C^{2}$ surfaces. Let $L_{1}$ and $L_{2}$ be lines in the $(x, y)$ plane, passing through $\left(x_{0}, y_{0}\right)$ in the (orthogonal) directions of principal curvature of the surface $z=$ $\Psi_{0}(x, y)$. Inside a sufficiently small neighborhood, $N$, of $\left(x_{0}, y_{0}\right), \Psi_{0}(x$, $y)<0$ at all points of $L_{1} \cap N$, and $\Psi_{0}(x, y)>0$ at all points of $L_{2} \cap$ $N$, except for the point $\left(x_{0}, y_{0}\right)$, where $\Psi_{0}(x, y)=0$.

The lines $L_{1}, L_{2}$, define a right-handed coordinate system. We denote by $l_{1}, l_{2}, l_{3}$, and $l_{4}$, the intersections of $N$ with the half-lines resulting from the deletion of $\left(x_{0}, y_{0}\right)$ from $L_{1}$ and $L_{2}$, as shown in Figure 1. 


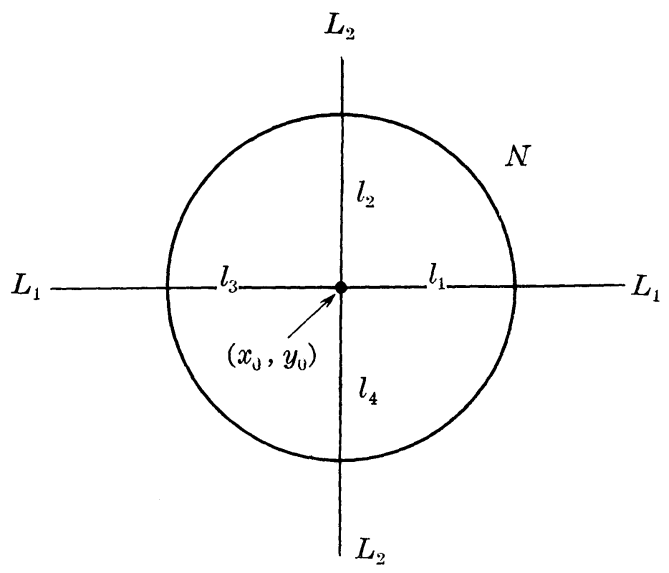

Figure 1

Thus, $\Psi_{0}(x, y)<0$ on $l_{1}, l_{3}$, and $>0$ on $l_{2}, l_{4}$, respectively.

Let $R_{0}$ denote the domain of definition of $\Psi_{0}(x, y) . R_{0}$ is the intersection of $R$ with the projection of $P_{0}$ on the $(x, y)$ plane, as illustrated by Figure 4. Since $R$ is a disk of radius $\rho<1 / H$, it follows that $\partial R_{0}$ is either a circle of radius $\rho$, or consists in part of a circle of radius $\rho$, and part of a circle of radius $1 / H$.

The open sets $G_{j}, j=1, \cdots 4$, are defined as follows:

$G_{j}$ is the largest connected open subset of $R_{0}$ containing $l_{j}$, and such that the sign of $\Psi_{0}(x, y)$ is everywhere the same in $G_{j}$.

LEMMA 3.2.

$$
G_{1} \cap G_{3} \text { and } G_{2} \cap G_{4} \text { are empty . }
$$

Proof. Let $\Gamma_{j}=\partial G_{j}, j=1, \cdots 4$.

Each set $\Gamma_{j}$ consists only of points where $\Psi_{0}(x, y)=0$, or of points of $\partial R_{0}$, where $\Psi_{0}(x, y)$ may or may not equal zero.

Since $\Psi_{0}(x, y)$ is the difference of two solutions of equation (1), it follows from Theorem 2.2 that $\Psi_{0}(x, y)$ cannot vanish identically on any of the boundary sets $\Gamma_{j}$, unless $\phi(x, y)$ is part of a hemisphere of radius $1 / H$. Since $\vec{x}_{0}$ is not an umbilical point, this is not the case.

If $\Psi_{0} \neq 0$ at some point $p_{j}$ of $\Gamma_{j}$, then $p_{j}$ cannot be an interior point of $R_{0}$, by the maximality of $G_{j}$. Therefore there exist points $p_{1} \cdots p_{4}$, such that

(i) $p_{j} \in \Gamma_{j} \cap \partial R_{0}, j=1, \cdots 4$

(ii) $\Psi_{0}<0$ on $p_{1}$ and $p_{3}, \Psi_{0}>0$ on $p_{2}, p_{4}$.

Let $g_{j} \in l_{j}, j=1, \cdots 4$, and let $p_{j}$ and $g_{j}$ be connected by the continuous curve $C_{j}$ lying entirely within $G_{j}$, for $j=1, \cdots 4$. 


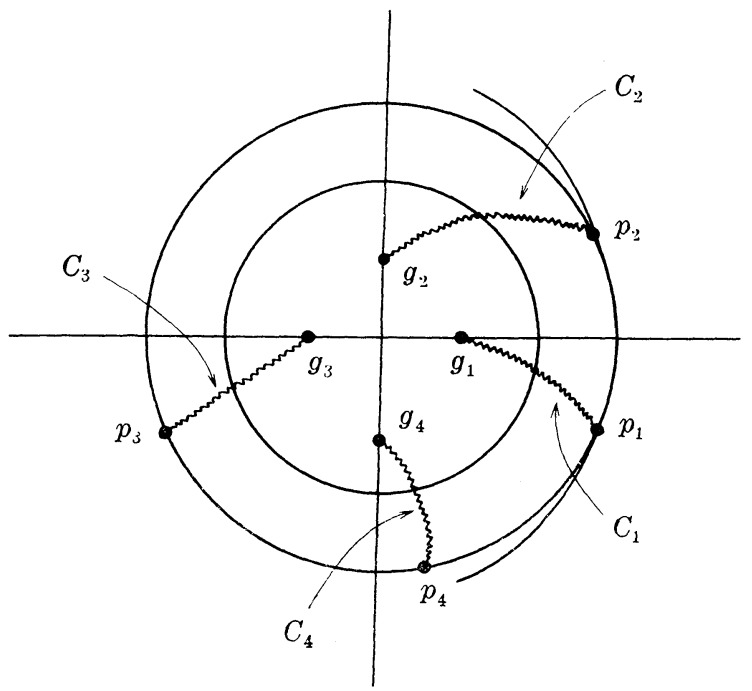

FIGURE 2

It follows that the sets $G_{j}, j=1, \cdots, 4$, are disjoint. If, $G_{1} \cap G_{3}$ is not empty, then the points $g_{1}$ and $g_{3}$ could be connected by a continuous curve lying entirely within the set $G_{1}=G_{1} \cap G_{3}=G_{3}$. This curve must intersect either $C_{2}$ or $C_{4}$, where $\Psi_{0}(x, y)>0$, and a contradiction results.

Let $T$ denote the space curve determined by the values of $\phi(x, y)$ on $\partial R$, as illustrated by Figure 4 . The proof of Theorem II is completed by considering two cases:

Case $I$. The projection of $P_{0}$ on the $(x, y)$ plane contains $R$.

In this case, $R_{0}=R$, and $\partial R_{0}$ is the circle of radius $\rho$. It follows from the continuity of $\Psi_{0}(x, y)$, together with the arguments presented in the proof of Lemma 3.2, that on any arc of $\partial R_{0}$ joining a pair of the points $p_{j}$, there must be a point on which $\Psi_{0}(x, y)=0$. Therefore, the space curve $T$ and the interior tangent sphere $P_{0}$ coincide at least four times.

Case II. $R_{0} \neq R$. In this case, $\partial R_{0}=S_{H} \cup S_{\rho}$, where $S_{H}$ and $S_{\rho}$ are arcs of circles of radius $1 / H$ and $\rho$, respectively.

Lemma 3.3. If $R_{0} \neq R$, then $\Gamma_{2}$ and $\Gamma_{4}$ contain points of $S_{\rho}$ where $\Psi_{0}(x, y)>0$.

Proof. Suppose the contrary. Then $\Gamma_{2}$ consists of points where $\Psi_{0}(x, y)=0$, and points on $S_{H}$ where $\Psi_{0}(x, y)>0$.

Let $\Gamma_{2}=\Gamma_{\alpha} \cup \Gamma_{\beta}$, where $\Gamma_{\alpha}=$ the closure of the set of points of 
$\Gamma_{2} \cap S_{H}$ where $\Psi_{0}(x, y)>0$, and $\Gamma_{\beta}=$ the closure of the remainder. In general, $\Gamma_{\alpha} \cap \Gamma_{\beta}$ will not be empty.

Let $\phi^{1}(x, y)=z_{0 L}(x, y)$ and $\phi^{2}(x, y)=\phi(x, y)$. We will verify that $\Gamma_{\alpha}, \Gamma_{\beta}, \phi^{1}$ and $\phi^{2}$ satisfy the hypotheses of Theorem 2.3 in $G_{2}$.

Hypothesis (i) is satisfied since both $\phi^{1}$ and $\phi^{2}$ are solutions of equation (1) in $R_{0}$, and therefore in $G_{2}$.

If $x_{\alpha}$ is an interior point of $\Gamma_{\alpha}$, then $\Psi_{0}(x, y)$ is $>0$ at $x_{\alpha}$. By continuity, $\exists \varepsilon<0 \ni \Psi_{0}(p)>0$ for all points $p \varepsilon R_{0} \ni\left\|p-x_{\alpha}\right\|<\varepsilon$, where $\left\|p-x_{\alpha}\right\|$ denotes the Euclidean distance of $p$ from $x_{\alpha}$. Therefore, $R_{0} \cap\left\{p \mid\left\|p-x_{\alpha}\right\|<\varepsilon\right\} \subset G_{2}$, by virtue of the maximal character of the connected open set $G_{2}$ of which $x_{\alpha}$ is a boundary point. Therefore, as illustrated by Figure $3, x_{\alpha}$ is the end point of a line segment $S_{\alpha}$ lying entirely within $G_{2}$, verifying hypothesis (ii).

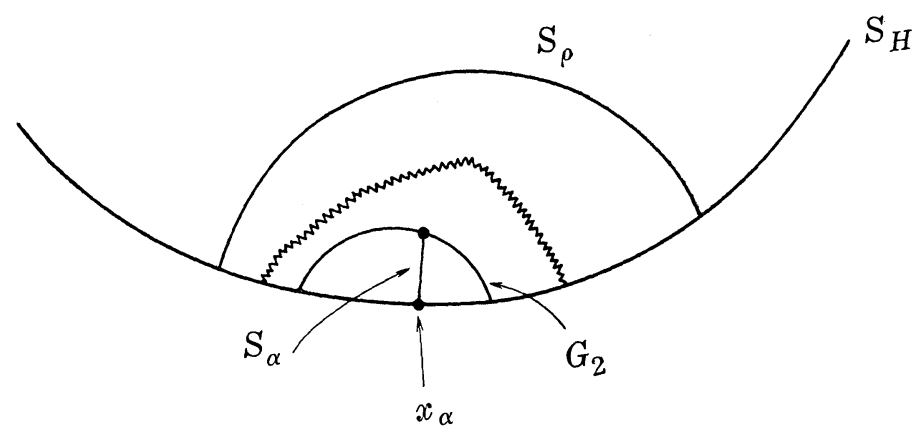

FIGURE 3

Since the gradient of $\phi^{1}(x, y)$ is infinite on $S_{H}$, hypothesis (iii) is verified.

By assumption, $\Psi_{0}(x, y) \equiv 0$ on $\Gamma_{\beta}$, so that $\phi^{1}=\phi^{2}$ on $\Gamma_{\beta}$. $Q\left(\phi^{1}\right)=$ $Q\left(\phi^{2}\right)$ in $G_{2}$, since $\dot{\phi}^{1}$ and $\dot{\phi}^{2}$ are solutions of equation (1). The conclusion of Theorem 2.3, that $\varliminf_{x \rightarrow \Gamma \alpha}\left[\phi^{1}-\phi_{2}\right] \geqq 0$, i.e., that $\Psi_{0}(x, y) \leqq 0$, completes the proof of Lemma 3.3 by contradiction. Thus, $\Gamma_{2}$ (and $\left.\Gamma_{4}\right)$ contain points of $S_{\rho}$ where $\Psi_{0}(x, y)>0$.

To complete the proof of Theorem II, let $p_{2}$ and $p_{4}$ be such points, i.e., $p_{j} \in \Gamma_{j} \cap S_{\rho}, \Psi_{0}\left(p_{j}\right)>0, j=2,4$.

By a brief argument, we can verify that there must exist a point $p_{3} \varepsilon \Gamma_{3} \cap S_{\rho}$, such that $\Psi_{0}<0$ on $p_{3}$, where $p_{3}$ lies between $p_{2}$ and $p_{4}$ on the arc $S_{\rho}$.

Thus,

$$
\begin{aligned}
\phi\left(p_{2}\right) & >z_{0 L}\left(p_{2}\right) \\
\phi\left(p_{3}\right) & <z_{0 L}\left(p_{3}\right) \\
\phi\left(p_{4}\right) & >z_{0 L}\left(p_{4}\right)
\end{aligned}
$$


Therefore, the space curve $T$ lies below the lower hemisphere of $P_{0}$ at the point $p_{3}$, and above the lower hemisphere of $P_{0}$ at the points $p_{2}$ and $p_{4}$.

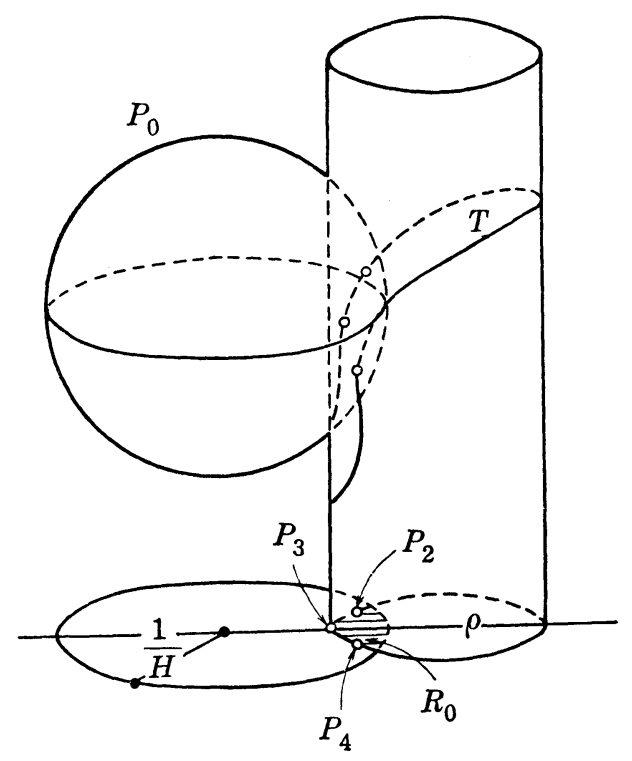

FIGURE 4

It follows that the space curve $T$ must have passed from the outside to the inside of $P_{0}$ at least twice. The points where the space curve has pierced $P_{0}$ must project onto $S_{\rho}$ at points which lie on $S_{\rho}$ between $p_{2}$ and $p_{4}$. The space curve $T$ cannot remain inside $P_{0}$, since there are points of $T$ which project onto the $(x, y)$ plane at points exterior to the projection on the $(x, y)$ plane of $P_{0}$. Therefore $T$ must eventually emerge from $P_{0}$, piercing $P_{0}$ twice more as it emerges.

We conclude that $T$ has at least four points in common with $P_{0}$.

4. Discussion. Theorem I can be used to derive a priori bounds on the gradient of solutions to the minimal surface equation. If $T$ satisfies the condition that the inclinations of all planes having at least three points in common with $T$ be uniformly bounded, then, an a priori bound on the inclinations of the tangent planes to the minimal surface $z=\phi(x, y)$ having $T$ for its boundary data, is immediately known.

Theorem II provides analogous bounds on the gradient of solutions of equation (1) with $H$ constant, $>0$. For example, Let $R$ be a disk of radius $\rho=(1-\varepsilon) / H, 0<\varepsilon<1$. Let $T$ satisfy the condition that for every sphere $P$ of radius $1 / H$ having four or more points in common with $T$, the projection on the $(x, y)$ plane of the center of $P$ is 
within $\varepsilon / 2 H$ of the center of $R$. It is easy to verify that under this assumption

$$
\sqrt{\phi_{x}^{2}+\phi_{y}^{2}} \leqq \sqrt{\frac{1-\varepsilon / 2}{1-(1-\varepsilon / 2)^{2}}}
$$

It is interesting to note that as $H \rightarrow 0$, the surfaces and interior tangent spheres of Theorem II become minimal surfaces and tangent planes, respectively, producing Theorem I, the three-point condition for minimal surfaces, as a limiting case.

\section{REFERENCES}

1. R. Courant and D. Hilbert, Methods of Mathematical Physics, Vol. II, Interscience Publishers, New York, 1962.

2. Robert Finn. Remarks relevant to minimal surfaces and to surfaces of prescribed mean curvature, Journal d'Analyse Mathematique, Vol. XIV, (1965), 139-160.

3. Tibor Radō, On the Problem of Plateau, Chelsea Publishing Co., New York, 1951,

Received November 23, 1970.

Naval Postgraduate School 



\title{
PACIFIC JOURNAL OF MATHEMATICS
}

\section{EDITORS}

\author{
H. SAMELSON \\ Stanford University \\ Stanford, California 94305 \\ C. R. HobBY \\ University of Washington \\ Seattle, Washington 98105
}

J. DugundJI

Department of Mathematics

University of Southern California

Los Angeles, California 90007

RICHARD ARENS

University of California

Los Angeles, California 90024

\section{ASSOCIATE EDITORS}
E. F. BECKENBACH
B. H. NeumanN
F. WOLF
K. YOSHIDA

\section{SUPPORTING INSTITUTIONS}

\author{
UNIVERSITY OF BRITISH COLUMBIA \\ CALIFORNIA INSTITUTE OF TECHNOLOGY \\ UNIVERSITY OF CALIFORNIA \\ MONTANA STATE UNIVERSITY \\ UNIVERSITY OF NEVADA \\ NEW MEXICO STATE UNIVERSITY \\ OREGON STATE UNIVERSITY \\ UNIVERSITY OF OREGON \\ OSAKA UNIVERSITY
}

\author{
UNIVERSITY OF SOUTHERN CALIFORNIA \\ STANFORD UNIVERSITY \\ UNIVERSITY OF TOKYO \\ UNIVERSITY OF UTAH \\ WASHINGTON STATE UNIVERSITY \\ UNIVERSITY OF WASHINGTON
AMERICAN MATHEMATICAL SOCIETY
NAVAL WEAPONS CENTER

The Supporting Institutions listed above contribute to the cost of publication of this Journal, but they are not owners or publishers and have no responsibility for its content or policies.

Mathematical papers intended for publication in the Pacific Journal of Mathematics should be in typed form or offset-reproduced, (not dittoed), double spaced with large margins. Underline Greek letters in red, German in green, and script in blue. The first paragraph or two must be capable of being used separately as a synopsis of the entire paper. The editorial "we" must not be used in the synopsis, and items of the bibliography should not be cited there unless absolutely necessary, in which case they must be identified by author and Journal, rather than by item number. Manuscripts, in duplicate if possible, may be sent to any one of the four editors. Please classify according to the scheme of Math. Rev. Index to Vol. 39. All other communications to the editors should be addressed to the managing editor, Richard Arens, University of California, Los Angeles, California, 90024.

50 reprints are provided free for each article; additional copies may be obtained at cost in multiples of 50 .

The Pacific Journal of Mathematics is published monthly. Effective with Volume 16 the price per volume (3 numbers) is $\$ 8.00$; single issues, $\$ 3.00$. Special price for current issues to individual faculty members of supporting institutions and to individual members of the American Mathematical Society: $\$ 4.00$ per volume; single issues $\$ 1.50$. Back numbers are available.

Subscriptions, orders for back numbers, and changes of address should be sent to Pacific Journal of Mathematics, 103 Highland Boulevard, Berkeley, California, 94708.

\section{PUBLISHED BY PACIFIC JOURNAL OF MATHEMATICS, A NON-PROFIT CORPORATION}

Printed at Kokusai Bunken Insatsusha (International Academic Printing Co., Ltd.), 270, 3chome Totsuka-cho, Shinjuku-ku, Tokyo 160, Japan. 


\section{Pacific Journal of Mathematics}

\section{Vol. 40, No. $2 \quad$ October, 1972}

Louis I. Alpert and L. V. Toralballa, An elementary definition of surface area in $E^{n+1}$ for smooth surfaces...........................

Eamon Boyd Barrett, A three point condition for surfaces of constant mean curvature........................................

Jan-Erik Björk, On the spectral radius formula in Banach algebras ....... 279

Peter Botta, Matrix inequalities and kernels of linear transformations . . . . 285

Bennett Eisenberg, Baxter's theorem and Varberg's conjecture ........... 291

Heinrich W. Guggenheimer, Approximation of curves .............. 301

A. Hedayat, An algebraic property of the totally symmetric loops associated with Kirkman-Steiner triple systems ....................... 305

Richard Howard Herman and Michael Charles Reed, Covariant representations of infinite tensor product algebras ................

Domingo Antonio Herrero, Analytic continuation of inner

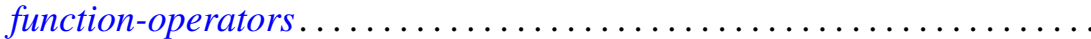

Franklin Lowenthal, Uniform finite generation of the affine group......... 341

Stephen H. McCleary, 0-primitive ordered permutation groups .......... 349

Malcolm Jay Sherman, Disjoint maximal invariant subspaces .......... 373

Mitsuru Nakai, Radon-Nikodým densities and Jacobians .............. 375

Mitsuru Nakai, Royden algebras and quasi-isometries of Riemannian manifolds. . .

Russell Daniel Rupp, Jr., A new type of variational theory sufficiency

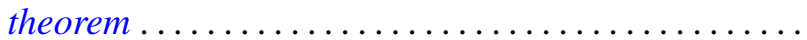

Helga Schirmer, Fixed point and coincidence sets of biconnected multifunctions on trees..........................

Murray Silver, On extremal figures admissible relative to rectangular

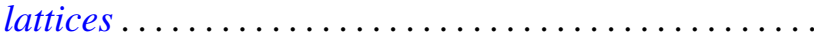

James DeWitt Stein, The open mapping theorem for spaces with unique segments ...

Arne Stray, Approximation and interpolation

Donald Curtis Taylor, A general Phillips theorem for $C^{*}$-algebras and some applications

Florian Vasilescu, On the operator $M(Y)=T Y S^{-1}$ in locally convex algebras...

Philip William Walker, Asymptotics for a class of weighted eigenvalue problems...

Kenneth S. Williams, Exponential sums over $\mathrm{GF}\left(2^{n}\right)$. 